\title{
Valor Prognóstico da Ecocardiografia com Estresse pela Dobutamina Associada à Atropina
}

\author{
W ilson M athias Jr, Ulisses Stella, Flávio Baruta, Adriana Cordovil, José Lázaro Andrade, A ntonio \\ Carlos Carvalho, Expedito Ribeiro, Renata Duprat, Eulógio Martinez
}

São Paulo, SP

\begin{abstract}
Objetivo - Avaliar o valor prognóstico para eventos cardíacos maiores da ecocardiografia com estresse pela dobutamina associada à atropina (EEDA).

Métodos - Estudados 452 pacientes consecutivos, com alto risco para presença de doença arterial coronária, acompanhados por um período médio de 23 meses.

Resultados - Houve 9 mortes cardíacas e 2 infartos agudos do miocárdio em pacientes com EEDA positiva e 2 infartos e 1 morte cardiaca em pacientes com EEDA negativa.

Conclusão - A EEDA é capaz de identificar pacientes de alto risco para eventos maiores.
\end{abstract}

Palavras-chave: ecocardiografia, coronariopatia, dobutamina

\section{Prognostic Value of Dobutamine-Atropine Stress Echocardiography}

Purpose - To determine the prognostic value of dobutamine-atropine stress echocardiography (DASE).

Methods - We studied 452 consecutive patients at high risk for coronary artery disease, using DASE. They were followed during a mean period of 23 months.

Results - There were 9 cardiac deaths and 2 acute myocardial infarctions in the group of patients with positive tests, and there were 2 myocardial infarctions and 1 cardiac death in patients with negative DASE.

Conclusion - We conclude that DASE reliably identifies patients at high risk for hard cardiac events.

Key-words: echocardiography, coronary artery disease, dobutamine

Arq Bras Cardiol, volume 69 (nº 2), 95-99, 1997

A doença arterial coronária (DAC) é responsável por mais de 1.500.000 infartos agudos do miocárdio (IAM) e por, aproximadamente, 550.000 mortes anuais nos Estados Unidos da América do Norte, perfazendo $25 \%$ de todos os óbitos deste país. Significante número desses óbitos é de indivíduos jovens e perto de $60 \%$ deles ocorrem de forma súbita na $1^{\mathrm{a}}$ hora após o início dos sintomas ${ }^{1}$. Por outro lado, de aproximadamente 1,7 milhões de pacientes internados, anualmente, por suspeita de IAM, somente em um terço deles o diagnósticoé confirmado ${ }^{2}$.

No Brasil, em 1989, morreram 64.000 pessoas devidoà DAC e, em 1993, aproximadamente 22.500 pessoas no Estado de São Paulo, respondendo por, aproximadamente, $33 \%$ de todas as mortes ${ }^{3}$.

Devido ao impacto social desta doença, a avaliação precoce da DAC tem despertado grande interesse ao longo do tempo.

Escola Paulista de Medicina-UNIFESP e Hospital Unicor - São Paulo Correspondência: Wilson Mathias Jr - Rua Itapeva, 500 - conj 5C - 01332-902 São Paulo, SP

Recebido para publicação em 11/4/97

Aceito em 11/6/97
Ainda mais importante do que analisar a acurácia diagnóstica de um exame para o diagnóstico de DAC é avaliar seu valor prognóstico para a previsão de IAM e morte de origem cardíaca, o que, em última análise, é a informação procurada pelo médico, a fim de identificar um subgrupo de indivíduos de alto risco, que poderiam obter maior benefício através do emprego de formas terapêuticas invasivas, como a angioplastia transluminal coronária (ATC) ou a revascularização do miocárdio ${ }^{4}$.

Ryan e col ${ }^{5}$ demonstraram que a ecocardiografia (ECO) com esforço é um método capaz de fornecer melhores informações prognósticas do que a ergometria. Estudaram 40 indivíduos, acompanhados de seis a 10 meses, avaliados do $10^{\circ}$ ao $21^{\circ}$ dia após o IAM, para a previsão de reinfarto, morte, angina e a necessidade para a realização de revascularização do miocárdio ou angioplastia. Houve $65 \%$ de especificidade e $55 \%$ de sensibilidade para a predição de eventos futuros pela ergometria e foram observadas $95 \%$ de especificidade e $80 \%$ de sensibilidade para a ECO com esforço. O valor prognóstico da ECO com esforço físico em esteira foi também avaliado em 115 pacientes com testes efi- 
cazes, máximos e negativos para isquemia, demonstrando que esses pacientes tiveram um bom prognóstico para a ausência de eventos em 23 meses $(98,2 \%)^{6}$.

No Brasil ${ }^{7}$, durante a seção brasileira de um estudo multicêntrico para a ECO com dipiridamol, EPIC (Echo Persantine International Cooperative Study), utilizando doses altas desta droga $(0,84 \mathrm{mg} / \mathrm{kg})$ em 43 pacientes no período após o IAM recente, demonstrou-se ser este um bom método na predição de eventos cardíacos futuros. Em outro estudo multicêntrico, foi avaliado o valor prognóstico da ECO com dipiridamol, após o IAM recente em estudo prospectivo em 925 pacientes. Óbito foi observado em $2 \%$ dos pacientes com teste negativo, em $4 \%$ com teste positivo, utilizando altas doses de dipiridamol e em $7 \%$ com teste positivo utilizando-se doses baixas de dipiridamol ${ }^{8}$.

O valor prognóstico da ECO pela dobutamina foi estudado em 51 pacientes com alta probabilidade de presença de DAC por um período médio de $24 \pm 9$ meses. No acompanhamento, observou-se um IAM, nove indivíduos apresentaram quadro clínico de angina instável e 13 foram submetidos à revascularização do miocárdio ou ATC. Alterações transitórias da contratilidade segmentar foram vistas em apenas $29 \%$ dos pacientes sem eventos e em $74 \%$ com eventos. IAM e angina instável ocorreram em oito (32\%) de 25 pacientes com teste positivo e em apenas dois (8\%) de 26 pacientes com teste negativo ${ }^{9}$.

Em outro estudo, agora através da ECO pela dobutamina associada à atropina, foram avaliados eventos cardíacos tardios em 430 indivíduos, com acompanhamento médio de $17 \pm 5$ meses. Ocorreram 79 eventos, sendo 11 mortes, 18 infartos agudos não fatais e 50 cirurgias de revascularização do miocárdio ou angioplastias. Pela análise de regressão logística, morte cardíaca foi predita pela idade e por alterações induzidas e transitórias da contratilidade segmentar do ventrículo esquerdo (VE) à ECO. Quando se somaram os eventos morte e IAM, estes foram preditos pela idade e história prévia de IAM e o ecocardiograma pela dobutamina não acrescentou novas informações. Estudando a combinação de todos os eventos (infarto, morte, revascularização do miocárdio e ATC), o aparecimento de alterações transitórias da contratilidade segmentar do VE, demonstradas pela ECO mostraram-se de maior poder prognóstico ${ }^{10}$.

Estes dois estudos que avaliam as implicações prognósticas em pacientes de alto risco, falham por incluírem na análise eventos menores, como a necessidade de revascularização do miocárdio ou angioplastia. Também não há nenhum estudo com ECO pela dobutamina avaliando o valor adicional da presença de isquemia sobre a função ventricular esquerda em repouso.

Nosso objetivo foi avaliar, o valor prognóstico independente e adicional da EEDA para prever eventos cardíacos futuros em 452 pacientes.

\section{Métodos}

No período de junho/91 a julho/93, 598 pacientes consecutivos com alta suspeita para a presença de corona- riopatia foram submetidos à EEDA e acompanhados no Hospital Unicor e Hospital São Paulo da EPM-UNIFESP, por um período médio de 23 meses. Após a análise dos testes, os resultados foram registrados em banco de dados utilizando planilha Excel da Microsoft com 114 campos de informação por paciente.

A administração endovenosa de dobutamina era controlada por bomba de infusão, com doses crescentes, em estágios com $3 \mathrm{~min}$ de duração, correspondentes a doses progressivas de $5 \mathrm{mcg} / \mathrm{kg} / \mathrm{min}$ até $40 \mathrm{mcg} / \mathrm{kg} / \mathrm{min}$. Caso até o $3^{\circ}$ minuto do estágio de $40 \mathrm{mcg} / \mathrm{kg} / \mathrm{min}$, o paciente não apresentasse sinais de isquemia miocárdica evidentes ao ecocardiograma e não tivesse atingido $85 \%$ da freqüência cardíaca(FC)máxima preditaparaaidade, atropina eraadministrada durante a infusão contínua de dobutamina, com doses de $0,25 \mathrm{mg}$ por minuto, até a dose máxima acumulativa de $1 \mathrm{mg}^{11}$. Havendo efeito colateral significativo ou isquemia miocárdica, $5 \mathrm{mg}$ de metoprolol eram injetados via endovenosa.

A cada estágio, determinavam-se a pressão arterial e a FC. A monitorização eletrocardiográfica era feita em três derivações contínuas durante o teste.

As imagens ecocardiográficas foram gravadas em fita VHS, utilizando-se um VCR-Panasonic e digitalizadas, lado a lado em equipamentos de digitalização de imagens disponíveis no mercado. O VE foi avaliado em quatro planos ecocardiográficos padrões: paraesternal longitudinal, paraesternal transversal ao nível dos músculos papilares e apical quatro e duas câmaras, divididos em 16 segmentos como recomendado pela Sociedade Americana de Ecocardiografia ${ }^{12}$.

Escores eram dados a cada um dos 16 segmentos do VEem repouso, em doses baixas de dobutamina $(5 \mathrm{e} 10 \mathrm{mcg} /$ $\mathrm{kg} / \mathrm{min}$ ) e no pico de infusão, sendo dado: valor 1 aos segmentos normais; 2 aos hipocinéticos; 3 aos acinéticos; e 4 aos discinéticos. O índice do escore de motilidade de parede (IEMP) era obtido através da soma dos escores dados a cada um dos 16 segmentos do VE divididos por 16.

O teste era interpretado como positivo para presença de isquemia sempre que aparecesse nova alteração na contratilidade regional do VE com a infusão de dobutamina, expresso pelo aumento no escore de ao menos um segmento do VE no mínimo em um ponto (por exemplo, escore $1 \mathrm{em}$ repouso e 2 no pico de infusão) com o aparecimento do sinal do "degrau", ou seja, afinamento abrupto da parede ventricular esquerda não observado antes do estresse. Também foi critério ecocardiográfico de isquemia quando havia piora de alteração contrátil preexistente (por exemplo, escore 2 em repouso e 3 no pico de infusão) ou quando havia alteração contrátil do VE em repouso (por exemplo, escore 3 ) que apresentasse melhora com doses baixas de dobutamina (por exemplo, escore 2) e posterior piora com doses altas (por exemplo, escore 3), indicando respectivamente viabilidade e isquemia miocárdica ${ }^{13}$.

Todos os ecocardiogramas com estresse foram avaliados após o término do teste em uma estação de trabalho por um investigador apenas. Em todas as análises citadas, o ecocardiografista desconhecia dados clínicos e de exames complementares dos pacientes. 
Foram analisados os primeiros 598 indivíduos com DAC conhecida ou altamente suspeita, submetidos à EEDA e definidos de acordo com probabilidade clínica $>50 \%$ de existência de DAC antes do teste ${ }^{14}$, calculada com base em sexo, idade e sintomas clínicos. Destes, 146 (24\%) foram perdidos no acompanhamento devido à mudança de endereço e ou telefone de contato, assim como ausência de dados para o acompanhamento nos prontuários hospitalares. Restaram 452 seguidos por um período médio de $23 \pm 12$ (mínimo de 1 mês e máximo de 46 meses, mediana de 21,4) meses.

Havia 145 mulheres e 307 homens. Neste grupo, a idade média foi de 57 anos, possuindo uma ou mais das características: tinham história de IAM prévio (145); haviam sido submetidos a procedimentos de revascularização do miocárdio (60); tinham ao menos dois fatores de risco para DAC (123) e dor precordial e ou teste ergométrico positivo para presença de isquemia (416).

Formulário de consentimento foi obtido em todos os pacientes e o projeto de pesquisa foi aprovado pelos comitês de ética do hospital São Paulo e hospital Unicor.

Eventos cardíacos analisados - menores: necessidade para revascularização do miocárdio ou angioplastia; maiores: óbito de origem cardíaca e IAM; totais: óbito de origem cardíaca, IAM, necessidade para revascularização do miocárdio e ATC.

Após a ocorrência de um evento, o acompanhamento clínico era encerrado para aquele paciente. Assim sendo, somente $\mathrm{o} 1^{\circ}$ evento ocorrido foi considerado. Os eventos foram confirmados por revisão de prontuários ou consulta médica. O diagnóstico de IAM sempre foi confirmado por um médico, com base nas características de dor precordial prolongada, eletrocardiograma (ECG) e enzimas sangüíneas (creatinofosfoquinase).

Avaliou-se a diferença estatística entre os pacientes com testes positivos e com testes negativos para isquemia em relação à ocorrência de eventos totais. Foi também analisado o valor adicional da positividade da EEDA em relação aos eventos totais por análise de regressão logística sobre as seguintes variáveis: sexo, idade $>75$ anos, presença de infarto prévio, revascularização do miocárdio ou angioplastia prévias, dor precordial, diabetes, mais de três fatores de risco para DAC, presença de ondas Q ao ECG, ou comprometimento da função ventricular esquerda em repouso e no pico de infusão (IEMP > 1,6) ao ecocardiograma.

Para a avaliação de eventos maiores com base na positividade ou negatividade dos testes, foram analisados 2 grupos: um de 198 pacientes com exames negativos e 70 pacientes com testes positivos que não foram submetidos a revascularização do miocárdio ou ATC. A probabilidade da ocorrência de eventos maiores no acompanhamento foi analisada em forma de curvas atuariais. Não houve diferença entre idade, sexo, revascularização miocárdica ou angioplastia ou disfunção ventricular esquerda severa (IEMP >2) entre os dois grupos.

A ocorrência de óbito no seguimento também foi analisada em pacientes com EEDA negativos e positivos com função ventricular preservada (IEMP $\leq 1,6$ ) e naqueles com função ventricular comprometida (IEMP > 1,6).

As curvas de sobrevida livre de eventos foram estudadas através de curvas atuariais pelo método de KaplanMayer e significância estatística dos eventos ocorridos no acompanhamento pelo Logrank Test. Para a avaliação de variáveis clínicas, eletrocardiográficas e ecocardiográficas na predição de eventos futuros, utilizou-se a análise de regressão logística pelo método stepwise de escolha de variáveis. $\mathrm{O}$ valor de $\mathrm{p}<0,05$ foi considerado estatisticamente significativo.

\section{Resultados}

A probabilidade média de presença de DAC antes da EEDA nos 452 pacientes analisados foi de $67,6 \%$.

Dos 452 pacientes acompanhados, houve $184(40,7 \%)$ eventos menores e $14(3,1 \%)$ eventos maiores (quatro IAM e 10 mortes de origem cardíaca). Restaram $254(56,2 \%)$ indivíduos que ficaram livres de eventos no acompanhamento.

Observamos $214(47,3 \%)$ com testes positivos para isquemia, sendo que $144(67,3 \%)$ tiveram eventos menores e $11(5,1 \%)$ eventos maiores (dois IAM e nove mortes de origem cardíaca). Houve 238 (52,7\%) indivíduos com teste negativo para isquemia, sendo que $40(16,8 \%)$ tiveram eventos menores e três $(1,3 \%)$ tiveram eventos maiores (uma morte e dois IAM), gerando significativa diferença entre os grupos de pacientes com testes positivos e negativos em relação a eventos totais $(\mathrm{p}<0,0001)$.

A análise das variáveis mais significativas na predição de eventos cardíacos futuros (eventos totais) foi feita através da análise de regressão logística. Observou-se que pacientes com positividade na EEDA tiveram 5,69 vezes mais chance de apresentar um evento cardíaco no acompanhamento do que os com testes negativos. Pacientes com DAC prévia tratada por angioplastia ou cirurgia de revascularização do miocárdio - marcadores de DAC grave - tiveram até três vezes mais chance, seguidas da presença de diabetes $(2,8)$, do IEMP no pico de infusão acima de 1,6 $(2,78)$ : marcador de presença de disfunção ventricular esquerda durante o estresse farmacológico, sexo masculino, $(1,83)$ e a presença de ondas Q noECG de repouso, (1,79).

A ocorrência de óbito foi significativamente maior nos indivíduos com testes positivos (9/70), quando comparados ao grupo com teste negativo para isquemia miocárdica (1/ 198), ( $\mathrm{p}=0,0068)$. A curva atuarial mostra a probabilidade de óbito em 46 meses de $25,6 \%$ para os pacientes com teste positivo quando comparados a $0,7 \%$ dos pacientes com teste negativo (fig. 1). Estudando-se somente a ocorrência de IAM entre os grupos com teste negativo para isquemia (2/ 198 pacientes) em relação aos com teste positivo para isquemia (2/70 pacientes), observa-se diferença estatística entre os dois grupos $(\mathrm{p}=0,0424)$. A curva atuarial demonstra probabilidade de IAM de 5,3\% para pacientes com teste positivo em relação a $1,4 \%$ dos pacientes com testes negati$\operatorname{vos}$ (fig. 2).

Dividindo-se os pacientes com teste negativo para 


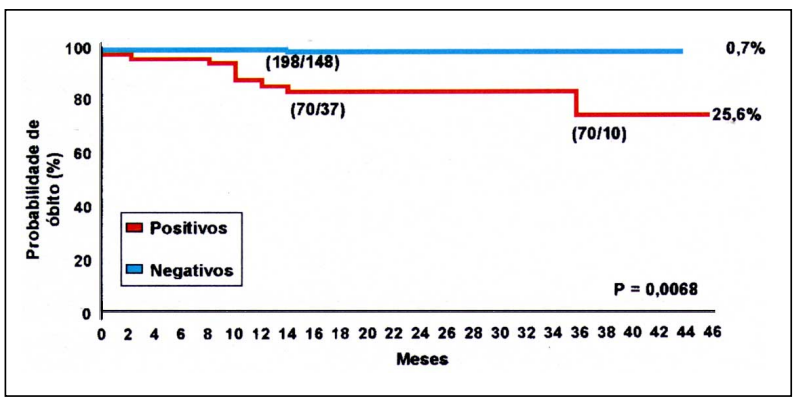

Fig. 1 - Curva atuarial demonstrando as probabilidades de óbito para pacientes com ecocardiografia com estresse pela dobutamina associada à atropina negativos e positivos para isquemia.

isquemia (198) em pacientes com função ventricular esquerda em repouso preservada (192), e pacientes com função ventricular comprometida (seis), nota-se a incidência de óbito no período de acompanhamento de $0 \%$ nos com função ventricular preservada e de $16,6 \%$ para os com função ventricular comprometida. Dividindo-se os indivíduos com teste positivo para isquemia (70) em pacientes com função ventricular esquerda em repouso preservada (50), e nos com função ventricular comprometida (20), nota-se incidência de óbito no período de acompanhamento de $6 \%$ naqueles com função ventricular preservada, e de $30 \%$ para os com função ventricular comprometida.

Houve diferença estatística entre todos os grupos analisados, exceto entre os pacientes com função ventricular esquerda comprometida (fig. 3 ).

\section{Discussão}

Dos 184 pacientes estudados submetidos à revascularização do miocárdio ou angioplastia no acompanhamento, após a EEDA, 144 (78\%) apresentaram testes positivos e somente $40(22 \%)$ testes negativos, o que destaca a importância deste exame na decisão clínica e reflete a alta acurácia diagnóstica em pacientes com DAC grave.

Neste estudo, observou-se significativa diferença na ocorrência de óbito no acompanhamento entre o grupo de pacientes com teste positivo em relação aos que apresenta-

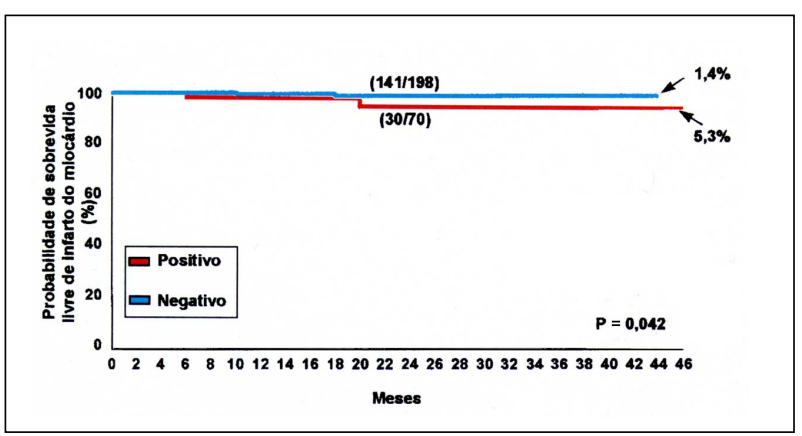

Fig. 2 - Curva atuarial demonstrando as probabilidades de ocorrência de infarto agudo do miocárdio para pacientes com ecocardiografia com estresse pela dobutamina associada à atropina negativos e positivos para isquemia.

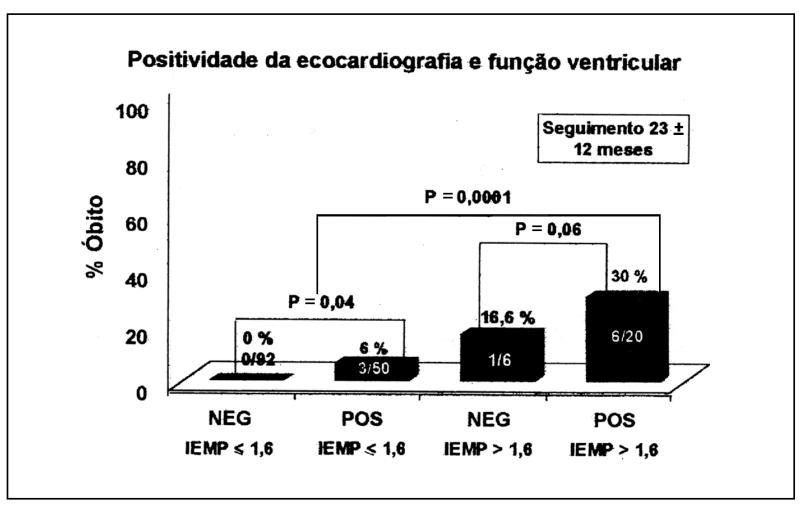

Fig. 3 - Comparação entre a ocorrência de óbito no seguimento médio de 23 meses em 268 pacientes com exames negativos (NEG) e positivos (POS), levando-se em consideração a função ventricular esquerda em repouso, demonstrada pelo índice do escore de motilidade de parede (IEMP).

vam teste negativo. Estes resultados são corroborados por autores com o uso da ECO com esforço ${ }^{5,6}$, pela ECO pelo dipiridamol ${ }^{7,8,15}$ e pela ECO pela dobutamina ${ }^{9,10}$.

Em nosso trabalho, o menor poder discriminativo para eventos futuros foi em relação à ocorrência de IAM com probabilidade de $1,4 \%$ para o grupo com teste negativo; enquanto que no grupo de pacientes com testes positivos, a probabilidade de infarto foi de 5,6\%. Estes resultados apresentaram a menor diferença entre os grupos para eventos maiores. Existem várias explicações para este fato: 1) $20 \%$ a $60 \%$ dos infartos do miocárdio podem ocorrer de forma silenciosa, sendo possível que alguns pacientes tenham tido este evento no período analisado e que o mesmo não tenha sido diagnosticado ${ }^{16,17}$; 2) a pequena diferença estatística entre os grupos, em relação à ocorrência de infarto pode estar relacionada à inabilidade de qualquer teste não invasivo para a pesquisa de isquemia identificar lesões coronárias discretas responsáveis por mais de $60 \%$ dos IAM $^{18}$. Dados recentes demonstram que pelas mesmas razões, a ECO com estresse não pode prever com boa acurácia diagnóstica a região de um futuro infarto do miocárdio ${ }^{19}$, fato também válido para outros métodos, como a cintilografia miocárdica de perfusão ${ }^{20,21}$.

A EEDA permitiu identificar, adequadamente, o grupo de pacientes com risco de morte. Possivelmente, isto se deve à excelente acurácia diagnóstica em pacientes com coronariopatia significativa, como aqueles com doença em três vasos.

Neste estudo, não houve óbito em indivíduos com função ventricular preservada e ausência de isquemia, enquanto houve $6 \%$ nos com função ventricular preservada e isquemia. Quando analisada a presença de disfunção ventricular esquerda associada à isquemia, observou-se ocorrência ainda maior (30\%). Estes dados são corroborados por estudos que demonstram que após o IAM, a presença de disfunção ventricular associada à isquemia aumentou de $4 \%$ para $11 \%$ a incidência de óbito no acompanhamento de 10 meses entre os pacientes que tinham ecocardiograma com estresse com dipiridamol positivo ${ }^{8}$.

A análise das variáveis clínicas, eletrocardiográficas e 
ecocardiográficas pelo método de regressão logística demonstrou que a positividade da ECO pela dobutamina associada à atropina foi o maior preditor da ocorrência de um evento no acompanhamento dos pacientes.

Todos estes resultados ratificam a superioridade da ECO na detecção dos sinais objetivos de isquemia miocárdica em relação aos dados clínicos e eletrocardiográficos na previsão de eventos cardíacos.

Em relação ao acompanhamento de pacientes, houve uma considerável descontinuidade (24\%), devido a razões alheias ao controle do pesquisador. Apesar desta significativa perda, o fato de não haver direcionamento ou tendências no acompanhamento faz pensar que a distribuição dos eventos tenha ocorrido de forma semelhante nesta população perdida. Outro fator limitante é a inclusão, na mesma análise de prognóstico de um grupo heterogêneo de indivíduos em diferentes condições clínicas, desde pacientes com angina até aqueles com grandes infartos de parede anterior.

Finalmente, é possível que o tratamento clínico medi- camentoso tenha sido intensificado na presença de resultados positivos, provavelmente, reduzindo a possibilidade de eventos. Os mesmos resultados positivos podem ter influenciado a ocorrência de procedimentos de revascularização do miocárdio, retirando assim, os pacientes de maior risco com testes positivos da análise de morte e infarto do miocárdio. Estas duas assertivas levam a vício na amostra, porém fortalecem a hipótese de que se os pacientes com testes positivos não tivessem sido medicados ou revascularizados, a diferença de eventos maiores observada entre os grupos de exames positivos e negativos poderia ser ainda maior.

Concluindo, a EEDA demonstrou ter excelente valor prognóstico para eventos cardíacos. Pacientes com teste negativo apresentaram grande probabilidade de ausência de eventos cardíacos em 46 meses. Pacientes com disfunção ventricular esquerda associada à isquemia miocárdica apresentaram significativa piora de seu prognóstico a longo prazo.

\section{Referências}

1. American Heart Association - 1990 Heart Facts. Dallas, American Heart Association National Center, $\mathrm{p} 1$.

2. National Center for Health Statistics - Utilization of short stay hospitals, United States, 1987. Vital Health Stat 1987; 31: 197.

3. Lotufo PA, Lolio CA - Tendência da mortalidade por doença isquêmica do coração no Estado de São Paulo: 1970 a 1989. Arq Bras Cardiol 1993; 61: 14953.

4. The Principal Investigators of CASS and Their Associates (The National Heart, Lung And Blood Institute) - Coronary Artery Surgery Study. Circulation 1981; 63(suppl I): I-12-13.

5. Ryan T, Armstrong WF, Feigembaum H-Risk stratification after acute myocardial infarction by means of exercise two dimensional echocardiography. Am Heart J 1987; 114: 1305-16.

6. Ismail G, LoE, Sada M et al - Long term prognosis of patients with a normal exercise echocardiogram and clinical suspicion of myocardial ischemia. Am J Cardiol 1995; 75: 934-5.

7. Camarano GP - Diagnóstico não invasivo da doença arterial coronária. A superioridade da ecocardiografia com dipiridamol. Arq Bras Cardiol 1990; 54: 239.

8. Picano E, Landi P, Bolognese L et al - On behalf of the EPIC study group - Prognostic value of dipyridamole echocardiography early after uncomplicated myocardial infarction: a large scale multicenter trial. Am J Med 1993; 608-18.

9. Mazeika PK, Nadzdin A, Oakley CM - Prognostic value of dobutamine stress echocardiography in patients with high pretest likehood of coronary artery disease. Am J Cardiol 1993; 71: 33-9.

10. Poldermans D, Fiorette PM, Boersma E et al - Dobutamine-atropine stress echocardiography and clinical data for predicting late cardiac events in patients with suspected coronary artery disease. Am J Med 1994; 97: 119-25.
11. MacNeill A, Fioretti PM, El Said EM et al. - Enhanced sensitivity for detection of coronary artery disease by addition of atropine to dobutamine echocardiography. Am J Cardiol 1992; 70: 41-6.

12. Shiller N, Shah P, Crawford Met al-Recommendations for quantitation of the left ventricle by two dimensional echocardiography. J Am Soc Echo 1989; 2: 358-67.

13. Picano E - Stress echocardiography. $2^{\text {nd }}$ ed. Springer-Verlag, 1994: 12-34.

14. Diamond GA, Forrester JS - Analysis of probability as an aid in the clinical diagnosis of coronary artery disease. N Engl J Med 1979; 300: 1350-8.

15. Severi S, Picano E, Lattanzi F et al - The diagnostic and prognostic value of dipyridamole echocardiography in patients with suspected coronary disease: a comparison with exercise electrocardiography. Circulation 1993; 89: 1160-73.

16. Margolis JL, Kannel WB, Feinleib M-Clinical features of unrecognized myocardial infarction: silent and symptomatic. Eighteen year follow-up. The Framingham Study. Am J Cardiol 1988; 32: 1.

17. Yano K, MaClean CJ - The incidence and prognosis of unrecognized myocardial infarction in the Honolulu, Hawai heart program. Arch Int Med 1989; 149: 1528.

18. Ambrose JA, Tannembaum MA, Alexopoulos Det al - Angiographic progression of coronary artery disease and the development of myocardial infarction. J Am Coll Cardiol 1988; 12: 56-62.

19. Varga A, Cortigiani L, Sicari R et al - Is stress echocardiography capable to predict the site of future myocardial infarction? J Am Coll Cardiol 1996; 28: 45-51.

20. Frais M, Botvinick E, Shosa D, O'Connel W-Are regions of ischemia detected on stress perfusion scintigraphy predictive of sites of subsequent myocardial infarctions? Br Heart J 1982; 47: 357-64.

21. Naqui T, Hachamovich R, Dev V, Shah PK-Does stress TC99m Sestamibi/Tl-201 myocardial SPECT predict the site of future acute myocardial infarction? Circulation 1995; 90: 545A. 